\title{
Anti-MAdCAM-I-Conjugated Nanocarriers Delivering Quantum Dots Enable Specific Imaging of Inflammatory Bowel Disease
}

This article was published in the following Dove Press journal: International Journal of Nanomedicine

\author{
Marta Truffi \\ Marta Sevieri iD ${ }^{2}$ \\ Lucia Morelli ${ }^{3}$ \\ Matteo Monieri (iD) ${ }^{2}$ \\ Serena Mazzucchelli iD ${ }^{2}$ \\ Luca Sorrentino ${ }^{2}$ \\ Raffaele Allevi \\ Arianna Bonizzi iD ${ }^{2}$ \\ Pietro Zerbi (iD) ${ }^{2}$ \\ Beatrice Marchini ${ }^{2}$ \\ Erika Longhi ${ }^{2}$ \\ Gianluca Matteo Sampietro (D) \\ Francesco Colombo iD 4 \\ Davide Prosperi iD $^{3}$ \\ Miriam Colombo ${ }^{3}$ \\ Fabio Corsi ${ }^{2,5}$ \\ 'Nanomedicine and Molecular Imaging \\ Laboratory, Istituti Clinici Scientifici \\ Maugeri IRCCS, Pavia 27100, Italy; \\ ${ }^{2}$ Department of Biomedical and Clinical \\ Sciences "Luigi Sacco, Università degli \\ studi di Milano, Milano, 20I57, Italy; \\ ${ }^{3}$ NanoBioLab, Department of \\ Biotechnology and Biosciences, \\ Università degli studi di Milano- \\ Bicocca, Milano, 20126, Italy; ${ }^{4}$ IBD \\ Surgery Unit, ASST Fatebenefratelli Sacco \\ - Ospedale "Luigi Sacco" Polo \\ Universitario, Milano, 20157, Italy; \\ ${ }^{5}$ Surgery Department, Breast Unit, Istituti \\ Clinici Scientifici Maugeri IRCCS, Pavia, \\ 27100 , Italy
}

Correspondence: Fabio Corsi Surgery Department, Breast Unit, Istituti Clinici Scientifici Maugeri IRCCS, via

Maugeri 4, Pavia 27100, Italy

Tel +3902503 I 9850

$\mathrm{Fax}+3902503$ I 9846

Email fabio.corsi@icsmaugeri.it
Purpose: Assessment of inflammatory bowel disease (IBD) currently relies on aspecific clinical signs of bowel inflammation. Specific imaging of the diseased bowel regions is still lacking. Here, we investigate mucosal addressin cell adhesion molecule 1 (MAdCAM-1) as a reliable and specific endothelial target for engineered nanoparticles delivering imaging agents to obtain an exact mapping of diseased bowel foci.

Materials and Methods: We generated a nanodevice composed of PLGA-PEG coupled with anti-MAdCAM-1 antibody half-chains and loaded with quantum dots (P@QD-MdC NPs). Bowel localization and systemic biodistribution of the nanoconjugate were analyzed upon injection in a murine model of chronic IBD obtained through repeated administration of dextran sulfate sodium salt. Specificity for diseased bowel regions was also assessed ex vivo in human specimens from patients with IBD. Potential for development as contrast agent in magnetic resonance imaging was assessed by preliminary study on animal model.

Results: Synthesized nanoparticles revealed good stability and monodispersity. Molecular targeting properties were analyzed in vitro in a cell culture model. Upon intravenous injection, P@QD-MdC NPs were localized in the bowel of colitic mice, with enhanced accumulation at $24 \mathrm{~h}$ post-injection compared to untargeted nanoparticles $(\mathrm{p}<0.05)$. Nanoparticles injection did not induce histologic lesions in non-target organs. Ex vivo exposure of human bowel specimens to $\mathrm{P} @ \mathrm{QD}-\mathrm{MdC}$ NPs revealed specific recognition of the diseased regions vs uninvolved tracts $(\mathrm{p}<0.0001)$. After loading with appropriate contrast agent, the nanoparticles enabled localized contrast enhancement of bowel mucosa in the rectum of treated mice.

Conclusion: P@QD-MdC NPs efficiently detected bowel inflammation foci, accurately following the expression pattern of MAdCAM-1. Fine-tuning of this nanoconjugate with appropriate imaging agents offers a promising non-invasive tool for specific IBD diagnosis. Keywords: polymeric nanoparticles, inflamed bowel, adhesion molecule, targeted imaging

\section{Introduction}

Inflammatory Bowel Disease (IBD) is a group of chronic relapsing conditions characterized by inflammation of the gastrointestinal tract. Its global incidence and prevalence are rising progressively. ${ }^{1-4}$ The two main types of IBD are Crohn's Disease and Ulcerative Colitis. ${ }^{5-7}$ Inflammation from Crohn's Disease can strike anywhere in the gastrointestinal tract, from mouth to anus. Patches of inflammation are interspersed between healthy portions of the gut and can penetrate the intestinal layers from inner to outer lining. Ulcerative Colitis is more localized 
than Crohn's Disease. Typically, the disease affects the large intestine including the rectum and anus, and only invades the inner lining of bowel tissue. Despite important distinctions, both Crohn's Disease and Ulcerative Colitis are characterized by an abnormal response of the immune system, and they may share some symptoms and some complications. Both are chronic and recurrent diseases, which present with periods of latency and exacerbation phases.

The clinical management of IBD is hindered by the complex and uncertain diagnosis, driven by a variety of aspecific and invasive exams and ultimately based on the clinical interpretation of endoscopic biopsies. ${ }^{8-10}$ Moreover, no definitive cure for these disorders is currently available - at best, therapy can control the disease, but patients have to undergo frequent tests to monitor the disease state and prevent or minimize the need for repeated surgical procedures. 5,11

The pathological process underlying IBD involves a dysregulated synthesis of pro-inflammatory cytokines, chronic recruitment of lymphocytes into the gutassociated lymphoid tissue, and the overexpression of adhesion molecules. $^{12,13}$ In particular, mucosal addressin cell adhesion molecule-1 (MAdCAM-1) has recently been recognized as a marker of IBD and has attracted the attention of clinicians and pharmaceutical companies as a promising therapeutic target for both Crohn's Disease and Ulcerative Colitis. ${ }^{14,15}$ This protein is mainly expressed on the surface of high endothelial venules of intestinal lymphoid tissues, where it binds the integrin $\alpha_{4}$ $\beta_{7}$ on gut-homing T-cells and mediates their migration into sites of inflammation in the underlying tissue. Increased expression of MAdCAM-1 on bowel microvascular endothelial cells correlates with disease activity while a decrease in expression correlates with response to therapy in IBD patients. ${ }^{16-18}$

Strategies that block leukocyte adhesion and traffic in IBD have raised high hopes for treatment of these diseases - an example is Vedolizumab (Entyvio ${ }^{\circledR}$, Takeda Pharmaceutical), an anti-integrin $\alpha_{4} \beta_{7}$ antibody, approved in 2014 by the FDA for the treatment of moderate-tosevere Ulcerative Colitis and Crohn's Disease. ${ }^{19-21}$ A fully human monoclonal antibody that binds to MAdCAM-1 (SHP647, previously PF-00547659) also represents a promising drug to selectively reduce lymphocyte homing to the intestinal tract. The Phase II TURANDOT trial showed that this antibody is well tolerated and enables remission in patients with moderate-to- severe Ulcerative Colitis. ${ }^{14,18}$ In the next years, the results from the Phase III studies will become available.

Beyond its potential as therapeutic target, MAdCAM-1 may represent an interesting target for molecular imaging of IBD in a way that is specific, reliable and early. ${ }^{22,23}$

In this study, we investigated MAdCAM-1 as a suitable target for engineered nanoparticles (NPs) loaded with imaging agents, in order to map bowel regions of vascular inflammation with precision. As a nanocarrier, we used polylactic-co-glycolic acid (PLGA), which is an excellent candidate for imaging purposes in that it is FDA-cleared, is biodegradable and has a low systemic toxicity. ${ }^{24,25}$ Moreover, the PLGA polymer may be quite easily conjugated with a variety of targeting molecules and it provides an extensive controlled release of drugs and imaging agents loaded inside, ${ }^{26}$ which results in time-dependent signal enhancement once the target organ is attained.

Recently, we generated PLGA micelles loaded with manganese oxide $(\mathrm{MnO}) \mathrm{NPs}$ and tested their potential as $\mathrm{T} 1$ contrast agents for magnetic resonance imaging (MRI). ${ }^{27}$ In the present study, we conjugated PLGA-PEG polymer micelles with the half-chain of a monoclonal MAdCAM-1 antibody and investigated the efficacy of the NPs' specificity for localization of IBD foci. To this end, the NPs were loaded with quantum dots (QDs) as a stable fluorescent tracer to easily follow nanolocalization in a preclinical experimental model of IBD. Targeting specificity of the produced nanodevice was also assessed ex vivo in human tissue by investigating the NPs' specificity for bowel lesions in samples from patients with Crohn's Disease. Finally, the imaging potential of MAdCAM-1-targeted PLGA-PEG polymer was assessed by preliminary MRI study on animal model.

The aim of this study was to assess the targeting efficacy and accuracy of PLGA-PEG polymer micelles functionalized with anti-MAdCAM-1 antibody derivatives, designed to enable specific detection of IBD regions by sustained molecularly targeted imaging.

\section{Materials and Methods \\ Materials}

Poly(D,L-lactide-co-glycolide) (Resomer ${ }^{\circledR}$ RG 504 H, 50:50 lactide:glycolide, acid terminal, MW 38.000-54.000 g/mol); Poly-vinyl alcohol (PVA, MW 9.000-10.000 g/mol, $80 \%$ hydrolyzed); N,N'-dicyclohexylcarbodiimide (DCC, MW $206.33 \mathrm{~g} / \mathrm{mol}, 99 \%$ ); Dimethylamino pyridine (DMAP, MW $122.17 \mathrm{~g} / \mathrm{mol}, \quad 99 \%$ ); N,N-Diisopropylethylamine 
(DIPEA, $129.24 \mathrm{~g} / \mathrm{mol}$ ); Ninhydrin (MW $178.14 \mathrm{~g} / \mathrm{mol}$ ); Ethanolamine (MW $61.08 \mathrm{~g} / \mathrm{mol}, \geq 98 \%$ ); $\mathrm{N}$, N-Dimethylformamide (DMF, anhydrous $\geq 99.8 \%$ ); 2,2 (Ethylenedioxy)bis(ethylamine) (EDBE, 98\%); and Dimethyl sulfoxide $148.20 \mathrm{~g} / \mathrm{mol}$ (DMSO, $\geq 99.9 \%$ ) were purchased from Sigma-Aldrich Co. (St Louis, MO, USA). Quantum Dot 800 ITK (QDs, Invitrogen) were obtained from Thermo Fisher Scientific. Chloroform ( $\geq 99.8 \%)$ was purchased from Honeywell Riedel-de-Haën ${ }^{\mathrm{TM}}$ (Germany). aMaleinimidohexanoic- $\omega-N H S$ PEG (Malhex-NH-PEG $-\mathrm{O}-\mathrm{C}_{3} \mathrm{H}_{6}$-CONHS, PEG-Mal, $3000 \mathrm{Da}$ ) was obtained from Rapp Polymer GmbH, Germany. Anti-MAdCAM1 antibody (AP-MAB0842, Rat Monoclonal, IgG2) was supplied by Abcam (Cambridge, UK).

\section{PLGA-PEG-Mal Synthesis}

For the polymer modification, the method used was as reported in a previous protocol with minor changes. ${ }^{28}$ Briefly, the polymer was dissolved in DMF $(15 \mathrm{~mL}$, $40 \mathrm{mg} / \mathrm{mL}$ ) with EDBE (molar ratio EDBE/PLGA= 10) and, after adding DCC (11 mg) and DMAP $(200 \mu \mathrm{g})$, the reaction was performed in a round-bottom flask $\mathrm{O} / \mathrm{N}$ (250 rpm, room temperature, under inert gas). Then, the solution was diluted with methanol, residual organic solvents were evaporated and PLGA-NH $\mathrm{N}_{2}$ was collected after precipitation $\left(20 \mathrm{~min}, 6200 \mathrm{rcf}, 4^{\circ} \mathrm{C}\right.$ ). Next, the conjugation with PEG-Mal (molar ratio PEG/PLGA = 5) was completed with DIPEA (molar ratio PEG/DIPEA = 1) in $10 \mathrm{~mL}$ of $\mathrm{CHCl}_{3} \mathrm{O} / \mathrm{N}$ (250 rpm, room temperature, under inert gas). In order to eliminate the unconjugated PEG, after the addition of methanol $(30 \mathrm{~mL})$, the polymer was isolated by precipitation twice. $^{29,30}$ The weight yield (50.91 $\left.\pm 6.50 \%\right)$ was calculated following the residual organic phase of evaporation under reduced pressure. The colorimetric ninhydrin assay (primary amine detection) was used to detect the degree of labelling: notably, $15 \mathrm{mg}$ of the product $\left(\mathrm{PLGA}-\mathrm{NH}_{2}\right)$ was solubilized in DMSO $(400 \mu \mathrm{L})$ then diluted with $100 \mu \mathrm{L}$ of ninhydrin solution $(3.5 \mathrm{mg} / \mathrm{mL})$. PLGA solution was used as a positive control; contrary ethanolamine solution $(18.75 \mathrm{mg} / \mathrm{mL})$ was used as the negative control. The samples were then incubated for 1 $\mathrm{h}$ at $65^{\circ} \mathrm{C}(400 \mathrm{rpm})$ and the colored conjugate was detected by UV-Vis spectroscopy (Spectrophotometer FluormaxHoriba Scientific, Italy) $(\lambda=600 \mathrm{~nm})$; the yield was calculated using a calibration curve of ethanolamine. The obtained degree of labelling for PLGA-NH $\mathrm{NH}_{2}$ was $71.90 \pm$ $12.70 \%$ and after binding of PEG there was no signal of primary amine detection.

\section{Synthesis of PLGA-PEG@QDs-HC-NPs}

PLGA-PEG@QDs were synthesized according to the single emulsion method, following a previous protocol. ${ }^{27,28}$ PLGAPEG-Mal solution (3 mg, $20 \mathrm{mg} / \mathrm{mL}$ in $\mathrm{CHCl}_{3}$ ), PLGA$\mathrm{COOH}\left(9 \mathrm{mg}, 12.85 \mathrm{mg} / \mathrm{mL}\right.$ in $\left.\mathrm{CHCl}_{3}\right)$ and QDs $(0.15$ nmol, $150 \mu \mathrm{L}$ in $\mathrm{CHCl}_{3}$ ) underwent double sonication with $40 \mathrm{~mL}$ of PVA $2 \%$ solution using a tip sonicator (Sonifier Sound, Branson Ultrasonics, Shanghai, People's Republic of China) for $30 \mathrm{~s}$ at $38 \%$ intensity in ice bath. The product was immediately poured to allow evaporation of the organic solvent ( $4 \mathrm{~h}$, room temperature, $750 \mathrm{rpm}$ ). After stirring, NPs were washed thrice with double-distilled water and collected by centrifugation at $19500 \mathrm{rcf}$, for $20 \mathrm{~min}$ at $4^{\circ} \mathrm{C}$ (Heraeus Fresco 21; Thermo Fisher Scientific, Göteborg, Sweden). The antibody binding was performed according to a previous protocol. ${ }^{31}$ Briefly, in order to reduce the disulfide bridges between the two heavy chains of the antibody, anti-MAdCAM1 or trastuzumab (TZ, Herceptin, Genentech) were dissolved in EDTA-PBS $(10 \mu \mathrm{g} / \mathrm{mL})$ and added to the 2-mercaptoethanolamine kit (MEA, Thermo Fisher Scientific). The obtained half-chain antibody portions (HC) were immediately added to NPs ( $1 \mathrm{mg}$ ) and incubated for $3 \mathrm{~h}$ at $4{ }^{\circ} \mathrm{C}$. At the end of the process, excess reagents were removed and NPs were collected by centrifugation (19500 rcf, $20 \mathrm{~min}, 4^{\circ} \mathrm{C}$ ) and freeze-dried in an Alpha 1-2 LD freeze dryer (Christ, Memmingen, Germany) at $0.500 \mathrm{mbar},-53^{\circ} \mathrm{C}$, $12 \mathrm{~h}$. The process yield was calculated as the ratio between NPs lyophilized and the initial raw materials.

\section{Dynamic Light Scattering and Zeta Potential Measurements}

Dynamic Light Scattering (DLS, Zetasizer Nano ZS; Malvern Instruments, Cambridge, UK) was selected to characterize NPs in terms of size, size distribution and Zeta potential; the analysis was performed in ultrapure water at $1 \mathrm{mg} / \mathrm{mL}$, at $25^{\circ} \mathrm{C}$. To calculate NPs' hydrodynamic diameter, the scattered light from the NPs in suspension was detected by the instrument considering medium viscosity. NPs size distribution was expressed by the polydispersity index (PDI) where PDI $\leq 0.2$ constitutes a monodisperse NP population.

\section{FTIR Spectroscopy}

Infrared absorbance measurements were performed using a Varian 610-IR infrared microscope associated to a Varian 670-IR Fourier-Transform Infrared (FTIR) spectrometer (Varian Australia Pty Ltd, Mulgrave VIC, Australia), 
with a high-sensitivity MCT detector (Mercury, Cadmium, Tellurium) cooled in liquid nitrogen. The spectral resolution was $2 \mathrm{~cm}^{-1}$ and the spectral range was from 4000 to $800 \mathrm{~cm}^{-1}$. The PLGA-COOH, PLGA-NH $\mathrm{N}_{2}$ and PLGAPEG-Mal (2 $\mathrm{mg}$ in $\mathrm{CHCl}_{3}$ ) samples were deposited on $\mathrm{BaF}_{2}$ plate and analyzed after solvent evaporation.

\section{Transmission Electron Microscopy}

PLGA-PEG@QDs were observed by Jeol JEM 1220, 200 $\mathrm{kV}$ transmission electron microscopy (TEM). The sample was prepared depositing the NPs suspension onto a formvar/carbon-coated 200-mesh copper grid (Ted Pella, CA, USA), negatively stained with $1 \%$ uranyl acetate and dried before examination.

For the analysis of nanoparticles in cell culture, cells $(9 \times$ $10^{5}$ ) were seeded on tissue culture plates and incubated with $0.3 \mathrm{mg} / \mathrm{mL}$ of PLGA-PEG-HC at $37^{\circ} \mathrm{C}$ for different periods of time (15 min or 1, 3, 24, $48 \mathrm{~h}$ ). At the end of incubation, cells were washed three times with PBS, detached and collected in eppendorf tubes for examination by TEM (Tecnai Spirit BioTWIN), as previously described. ${ }^{31}$

\section{Cell Binding Assay}

HER2 overexpressing SKBR3 cell line was purchased from ATCC-LGC Standards and cultured in Dulbecco's Modified Eagle Medium and Ham's F12 Nutrient Mixture (50\% v/v), plus $10 \%$ heat inactivated fetal bovine serum, $2 \mathrm{mM}$ L-glutamine, $100 \mathrm{U} / \mathrm{mL}$ penicillin, and $0.1 \mathrm{mg} / \mathrm{mL}$ streptomycin (Euroclone). Cells were maintained at $37^{\circ} \mathrm{C}$ in a humidified atmosphere with $5 \% \mathrm{CO}_{2}$. For the binding assay, cells were transferred into plastic tubes $\left(5 \times 10^{5}\right.$ cells/tube) and incubated at $4{ }^{\circ} \mathrm{C}$ for $2 \mathrm{~h}$ in PBS with $0.3 \%$ bovine serum albumin (BSA) and $0.3 \mathrm{mg} / \mathrm{mL}$ of PLGA-HC or PLGA-IgG NPs or a corresponding concentration of free trastuzumab antibody. Cells were washed three times with PBS, stained with Alexa Fluor-488-conjugated secondary antibody and analyzed by CytoFLEX flow cytometer (Beckman Coulter), as previously reported. ${ }^{31}$

\section{Immunofluorescence}

Cells $\left(0.5 \times 10^{5}\right)$ were plated on glass coverslips in a 24-well plate and incubated with $0.3 \mathrm{mg} / \mathrm{mL}$ of PLGA-HC at $37^{\circ} \mathrm{C}$. After the indicated incubation time, cells were washed three times with PBS and treated for HER2 immunostaining as previously described. ${ }^{31}$ Anti-HER2 antibody and PLGA-HC were revealed with Alexa Fluor-546- and Alexa Fluor-488conjugated secondary antibody (1:300, Thermo Fisher Scientific), respectively.

\section{Animal Model of Colitis}

C57BL/6 mice (female, 6 weeks old) were supplied by Charles River, group housed (4 mice/cage) under standard laboratory conditions and allowed to acclimatize for 1 week. Dextran sulfate sodium salt (DSS, 2.5\% w/v, $40 \mathrm{kDa}$, MP Biomedicals) was dissolved in drinking water and administered ad libitum to mice for three cycles ( 5 days DSS followed by 7 days of tap water), as previously described. ${ }^{32}$ The beverage was replaced by fresh solution every 2 days. The control group received tap water only. Colitis was scored daily using standard parameters that included body weight change (scored on a $0-6$ scale as: $0=$ no loss, $1=1-5 \%$ loss, $2=5-10 \%$ loss, $3=10-15 \%$ loss, $4=15-20 \%$ loss, $5=$ $20-25 \%$ loss, $6>25 \%$ loss), stool consistency (scored on a $0-4$ scale as: $0=$ normal, $1=$ soft with pellets, $2=$ soft without pellets, $4=$ diarrhea) and presence of blood in the stools (scored on a $0-2$ scale, as: $0=$ absence, $1=$ mild, $2=$ severe) were monitored daily. A disease activity index (DAI) was calculated as sum of the individual scores, as reported. ${ }^{33}$ At the end of treatment (day 36), bowel was dissected from mice and cleared from fecal material through gentle flushing with PBS.

Colon length was measured from the ileum-colon boundary down to the rectum. Dissected bowels were rolled and fixed in 10\% buffered formalin solution for histopathological assessment.

\section{NPs Administration}

Mice previously treated with DSS were randomly divided into four groups $(\mathrm{n}=23$ each) as group A: control (injected with vehicle only), group B: MAdCAM-1-targeted NPs (P@QD-MdC), group C: aspecific IgGconjugated NPs (P@QD-IgG), group D: bare NPs (P@QD). NPs were suspended in saline solution containing $0.9 \% \mathrm{NaCl}$ and administered as a single dose of $25 \mathrm{mg}$ PLGA $/ \mathrm{Kg}$ body weight by injection into the tail vein. NPs or vehicle were injected at the end of the third DSS cycle (day 36) upon gas anesthesia (2.5\% isoflurane). A total of 20 mice per group were dedicated to biodistribution analysis. Three additional mice per group were euthanized at $24 \mathrm{~h}$ post-injection and used for histopathological analysis.

\section{Tissue Samples Preparation and Fluorescence Analysis}

At $10 \mathrm{~min}, 1,6$ and $24 \mathrm{~h}$ post-NP-injection, 5 mice per group were euthanized for biodistribution analysis. Colon, small bowel, heart, spleen, lungs, kidneys, liver and brain 
were dissected, washed with PBS, wiped and arranged on a black sheet for image acquisition by an IVIS Lumina II system (Calipers Life Sciences) set at 430-500 nm excitation and equipped with an indocyanine green emission filter (720-840 nm). After the acquisition, images were corrected by spectral unmixing. Epifluorescence intensity of each organ was determined upon drawing appropriate regions of interest comprising each organ separately. NPs accumulation in the colon was measured as total epifluorescence collected from the ileocecal junction to the rectum. For each organ, average epifluorescence measured in control mice was subtracted as a reference for tissue autofluorescence.

At each time point, before euthanasia, blood was collected from the retro-orbital plexus of anesthetized mice and collected in EDTA-coated tubes (Becton Dickinson). Plasma was prepared by centrifugation at $1500 \times \mathrm{g}$ for $15 \mathrm{~min}$ at $4{ }^{\circ} \mathrm{C}$ and analyzed by spectrofluorimetry (Horiba; $\lambda_{\mathrm{ex}}=355 \mathrm{~nm} ; \lambda_{\mathrm{em}}=769 \mathrm{~nm}$ ). Background plasma fluorescence from control mice was subtracted from each sample.

\section{Histopathological Assessment}

Organ samples were fixed for $48 \mathrm{~h}$ in buffered formalin $(10 \%)$, embedded in paraffin and stained with hematoxylin and eosin (H\&E) before blinded examination.

To evaluate the histomorphology of intestinal inflammation, mice treated with NPs were scored individually by a blinded observer for density of inflammatory cell infiltrate $(0=$ no inflammation, $1=$ mild cell infiltrate, $2=$ moderate cell infiltrate, 3 = severe cell infiltrate); extent of leukocyte infiltration $(1=$ mucosal, $2=$ mucosal + submucosal, $3=$ transmural $)$; granulocyte component $(1=0-10 \%, 2=>10 \%$ focal, $3=$ $>10 \%$ diffuse); and epithelial changes $(0=$ normal morphology, 1 = epithelial regeneration, $2=$ epithelial erosion and regeneration, $3=$ ulceration). The different parameters were summed to obtain the total histologic score (0-12 range), as adapted from previous reports. ${ }^{34,35}$

\section{Immunohistochemistry}

Three micrometer paraffin sections of colon samples from mice were cut and treated for anti-MAdCAM-1 immunostaining, as previously reported. ${ }^{22}$

For immunohistochemistry on human tissue samples, after deparaffinization and endogenous peroxidase blockade, antigen retrieval was performed by microwave in 0.01 M EDTA buffer. Anti-human MAdCAM-1 monoclonal antibody (1:100; 314G8 clone, LS-Bio, Seattle, WA) was incubated for $1.5 \mathrm{~h}$ at room temperature. Slides were washed with Tris Buffer Solution and incubated with a MACH 4 Universal HRP-polymer kit (Biocare Medical, Concord, CA, USA). Peroxidase activity was visualized with DAB. Slides were counterstained with hematoxylin.

\section{Scanning Electron Microscopy}

NPs were sonicated in water, placed as a drop on a glass coverslip and air dried. After coating with gold-palladium, samples were examined with a scanning electron microscope (SEM, Leica S420).

For ultrastructural analysis of bowel tissue, a fragment from the proximal part of the colon and one from the distal part of the colon were collected from 2 mice at $24 \mathrm{~h}$ post-NP-injection. Tissue samples were fixed in $2.5 \%$ buffered glutaraldehyde, washed and postfixed in $1.5 \%$ buffered $\mathrm{OsO}_{4}$ at $4{ }^{\circ} \mathrm{C}$ for $2 \mathrm{~h}$, dehydrated in graded ethanol series and embedded in Epon 812. Semithin sections of $1.5 / 2.0 \mu \mathrm{m}$ were placed on a coverslip, treated with $2 \%$ sodium ethoxide for $15 \mathrm{~min}$, washed with ethyl alcohol and dried $\mathrm{O} / \mathrm{N}$ in hexamethyldisilazane. After coating with gold-palladium, samples were analyzed by SEM.

\section{Human Bowel Tissue Specimens}

Human bowel samples were collected from three patients with Crohn's Disease referred to the IBD Surgical Unit of ASST Fatebenefratelli-Sacco, "Luigi Sacco" University Hospital (Milano, Italy), a tertiary referral center for IBD management. Inclusion criteria were: age between 20 and 70 years old, proven diagnosis of Crohn's Disease, indication for ileal resection and preoperative staging by bowel ultrasound and abdominal MRI. Mean age of the patients included in the study was 44.4 years $( \pm 15.4)$.

From surgical resection, bowel tissue specimens were prepared by the pathologist in order to obtain fullthickness biopsies $\left(1 \mathrm{~cm}^{2}\right.$ each) from the stenotic diseased segment and the non-stenotic tract near the proximal resection margin. From stenotic and non-stenotic bowel tract, two adjacent biopsy samples were collected: one was covered with optimal cutting temperature compound (OCT, VWR International PBI Srl) for immediate freezing and NPs experiment, the other was fixed in buffered formalin $(10 \%)$ for histological assessment. 


\section{NPs for Validation on Human Samples}

PLGA-PEG NPs were conjugated with half-chains of a human MAdCAM-1 monoclonal antibody (314G8 clone, LS-Bio) and loaded with QDs as described above. Lyophilized NPs were resuspended in sterile double distilled water at $10 \mathrm{mg} / \mathrm{mL}$ and then diluted to the working concentration immediately prior to usage.

Human bowel cryosections were sliced (10 $\mu$ m-thick), fixed for $5 \mathrm{~min}$ in cold acetone, air dried for $10 \mathrm{~min}$ and rehydrated with PBS for $5 \mathrm{~min}$. Then, tissues were incubated with P@QD-MdC $(0.3 \mathrm{mg} / \mathrm{mL})$ for $12 \mathrm{~h}$ in PBS 1\% BSA at $4^{\circ} \mathrm{C}$. To visualize the cells in the tissue, bowel cryosections were stained with Alexa Fluor-488-conjugated Wheat Germ Agglutinin (WGA, 1:200 dilution, ThermoFisher Scientific) for $10 \mathrm{~min}$ at room temperature. For MAdCAM-1 costaining, before NPs incubation, tissues were blocked for $2 \mathrm{~h}$ in PBS with $2 \% \mathrm{FBS} / 2 \%$ goat serum, and incubated for $2 \mathrm{~h}$ with anti-MAdCAM1 antibody (1:100, 314G8 clone, LSBio) and then for $2 \mathrm{~h}$ with anti-mouse Alexa Fluor-488conjugated secondary antibody. After incubation, tissues were washed three times with PBS, slides were mounted in Dako mounting medium and images were acquired under the Leica SP8 system with $20 \times$ magnification lens. To visualize QDs fluorescence, excitation and emission were set at 405 $\mathrm{nm}$ and $800 \mathrm{~nm}$, respectively, while Alexa Fluor-488 fluorescence was recorded with a $488 \mathrm{~nm}$ excitation laser. Image analysis was performed with Fiji software.

\section{MRI Setup}

MnO-trapped PLGA-PEG NPs (MnO@PLGA) were prepared, as previously reported, ${ }^{27}$ and functionalized with the half-chain portions of anti-MAdCAM-1 antibody (APMAB0842, Abcam) as described above. NPs were suspended in saline and injected in DSS-treated mice by the tail vein (150 $\mu \mathrm{g} \mathrm{MnO} /$ mouse, infusion rate $100 \mu \mathrm{L} / \mathrm{min})$. At $24 \mathrm{~h}$ post-injection, MRI scan of the rectum was performed upon gas anesthesia ( $2.5 \%$ isoflurane) using a $7 \mathrm{~T}$ small-bore preclinical magnetic resonance scanner equipped with a mouse body volume-coil (BRUKER, BioSpec 70/30 USR, Paravision 5.1) and 450/675 mT/m gradients (slew-rate: $3400-4500 \mathrm{~T} / \mathrm{m} / \mathrm{s}$; rise-time $140 \mu \mathrm{s}$ ). MRI protocol and sequences are described as $\underline{\text { Supplementary Information. }}$

\section{Statistical Analysis}

Statistical analyses were conducted using two-tailed Student's $t$-test in case of data that passed the Shapiro-Wilk normality test, or with the non-parametric Wilcoxon-Mann-Whitney test in case of non-normal distribution of the data. For multigroup comparisons, the Kruskal-Wallis test was also applied. Results are expressed as mean \pm standard error (SE) or standard deviation (SD). Unless otherwise specified, the statistical significance threshold was set at $\mathrm{p}<0.05$.

\section{Ethical Approval}

Experimental protocols used for animal research went through the Ethics Committee for Animal Experimentation (OPBA) of the Università degli studi di Milano, according to institutional guidelines, and were subsequently approved by the Italian Ministry of Health (General Direction for Animal Health and Veterinary Medicines) prior to the commencement of the study (Authorization number 97/2018-PR). All experiments were performed following relevant institutional guidelines and according to national guidelines and regulations.

Collection and use of human bowel tissue samples were authorized by the Ethics Committee of ASST Fatebenefratelli Sacco (Milano Area 1) as protocol number 24916/2019. All patients signed a written informed consent prior to their inclusion in the study. Investigations were conducted in compliance with the ethical standards established by the Declaration of Helsinki and according to national guidelines and regulations. The study was approved by the authors' institutional review board.

\section{Results}

\section{NPs Synthesis and Characterization}

The protocol for polymer modification was established and PLGA-PEG-Mal was scanned with FTIR analysis to highlight the conjugation of PEG across the PLGA-NH $\mathrm{N}_{2}$ spectrum (Supplementary Figure 1). From it, NPs of PLGA@QDs with controlled size and homogenous size distributions were obtained via the single emulsion method (Table 1). At DLS analysis, and confirmed by dot blot (Supplementary Figure 2), the conjugation with anti-MAdCAM1 antibody half-chains determined a slight increase in the size of NPs which, upon further characterization by electron microscopy, appeared spherical shaped and around $150 \mathrm{~nm}$ in size with several QDs situated within the inner core of the PLGA polymer micelles (Figure 1 and Supplementary Figure 3). The discrepancy between the large size obtained with DLS analysis and TEM analysis could be the result of swelling effect of NPs resuspended in water. ${ }^{36}$ 
Table I NPs Characterization Measured by DLS Analysis and Process Yield. Data Represent Mean \pm SD $(n=3)$

\begin{tabular}{|l|l|l|l|l|}
\hline & Size (nm \pm SD) & PDI & Z-Potential (mV \pm SD) & Process Yield (\%, w/w \pm SD) \\
\hline PLGA-PEG@QDs & $197.9 \pm 10.1$ & $0.131 \pm 0.020$ & $-29.8 \pm 0.35$ & $56.1 \pm 13.4$ \\
PLGA-PEG@QDs-hlgG & $204.5 \pm 7.65$ & $0.147 \pm 0.030$ & $-31.2 \pm 0.63$ & $39.6 \pm 5.2$ \\
PLGA-PEG@QDs-hMdC & $209.7 \pm 17.5$ & $0.136 \pm 0.039$ & $-32.3 \pm 1.71$ & $37.5 \pm 7.3$ \\
\hline
\end{tabular}

Quantitation of QDs was determined by fluorescence spectrometry ( $\lambda_{\text {ex }} 300 \mathrm{~nm} ; \lambda_{\text {em }} 772 \mathrm{~nm}$, in $\mathrm{CHCl}_{3}$ ) and the encapsulation efficiency was determined using a calibration curve $(37.48 \% \pm 12.45, \mathrm{n}=3)$. The fluorescence signal of QDs was reproducible in three independent syntheses and after $24 \mathrm{~h}$ in PBS (pH 7.2) there was no decrease of intensity (Supplementary Figure 4).

\section{Targeting Properties of HC-Functionalized PLGA}

Since there is no proven robust cellular model that naturally overexpresses MAdCAM-1 protein, to study the molecular targeting properties of half-chain-decorated NPs, PLGAPEG-Mal were conjugated with the half-chain $(\mathrm{HC})$ portions of the well-known anti-HER2 monoclonal antibody trastuzumab. This allowed us to conduct proof of principle studies on the biological interaction of HC-nanoconjugates with appropriate cellular models grown in vitro. PLGA-PEG-HC rapidly and efficiently bound HER2-overexpressing cells ( $85.9 \pm 7.9 \%$ binding) as opposed to NPs coupled with HC portions of non-specific immunoglobulin (PLGA-PEG-IgG, $\mathrm{p}=0.02$, Figure $2 \mathrm{~A}$ ). Conjugation of $\mathrm{HC}$ portions to polymeric NPs yielded better results than conjugation of whole antibodies, as demonstrated by optimal preservation of target recognition specificity due to naturally oriented HC immobilization (Supplementary Figure 5). Increasing percentage of PEG-Mal did not significantly modify the final conjugation efficiency (Supplementary Figure 6), which probably reflects a maximal loading capacity due to steric hindrance of $\mathrm{HC}$ derivatives. Once bound to the molecular target,
PLGA-PEG-HC underwent receptor-mediated endocytosis, as shown by colocalization with the HER 2 target over time in confocal microscopy (Figure 2B). Endosomal compartmentalization of NPs was confirmed by TEM analysis. Then, progressive long-term degradation of the PLGA matrix occurred in the intracellular compartment within lysosomal vesicles after 48 hours of incubation, according to the biodegradation profile of the nanomaterial (Figure 2C).

\section{Experimental Model of Chronic Colitis}

A pre-clinical murine model of IBD was established by treating mice with three sequential cycles of $2.5 \%$ DSS in drinking water. During the first cycle of treatment, DSS administration induced significant body weight loss and softening of the stools, which was partially recovered during the second and third cycles of treatment (Figure 3A and B). Traces of fecal blood appeared upon DSS treatment but diminished during periods of recovery with tap water (Figure 3C). The overall disease activity index reached a peak score of 6.1 during the first cycle of DSS, but declined over time to settle at $<1$ at the end of treatment, in line with the chronic rather than acute behavior of colon inflammation at the end of the treatment (Figure 3D). Despite restrained clinical signs of the disease, DSS-treated mice showed significant reduction of colon length as compared to control mice (Figure 3E). Moreover, colon damage was clearly assessed by histology at the end of treatment: mice receiving DSS showed signs of chronic inflammation, characterized by increased infiltrate of lymphocytes and plasma cells, alteration of crypt architecture, and an alternated presence of
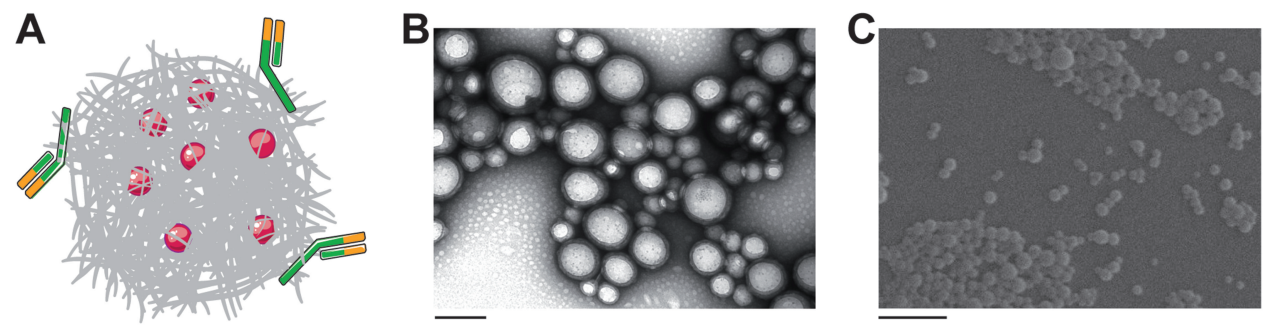

Figure I PLGA-PEG loaded with QDs and engineered with multiple half-chains of anti-MAdCAM-I antibody are shown as a schematic representation (A), TEM image (B, scale bar: $200 \mathrm{~nm}$ ) and SEM image (C, scale bar: I $\mu \mathrm{m})$. 


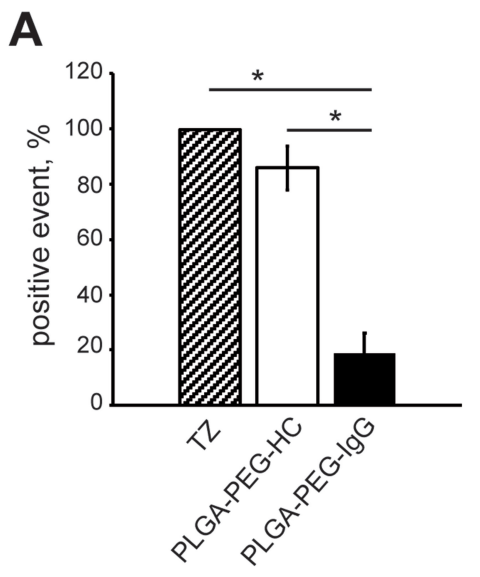

B

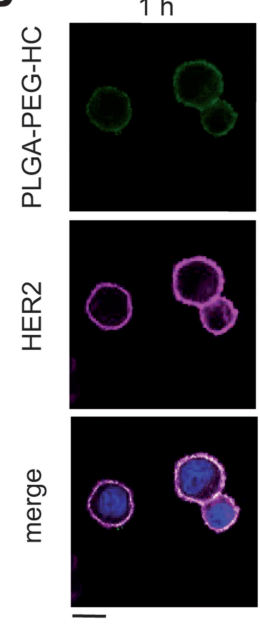

$3 \mathrm{~h}$
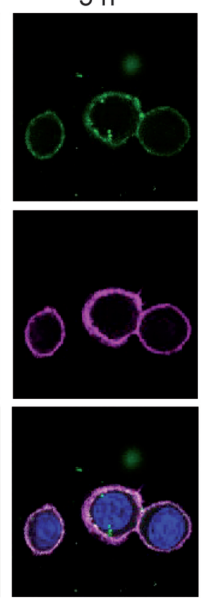

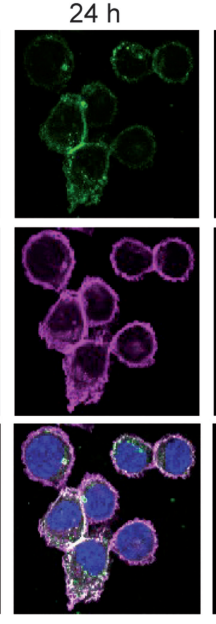

$48 \mathrm{~h}$

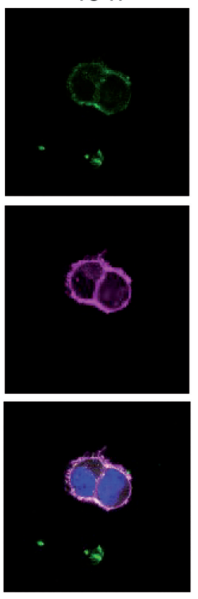

C

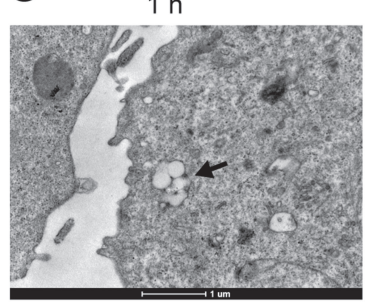

$3 \mathrm{~h}$

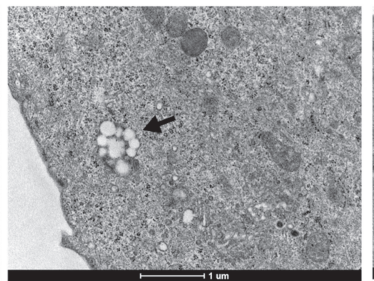

$24 \mathrm{~h}$

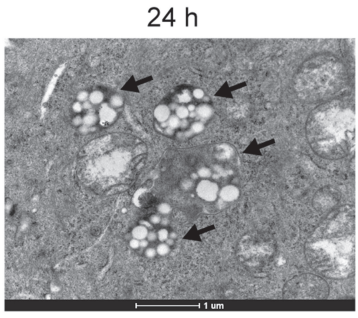

Figure 2 PLGA-PEG-HC holds targeting properties in cell culture. (A) Binding of PLGA-PEG-HC or aspecific PLGA-PEG-IgG NPs to SKBR3 cells. Incubation with corresponding concentration of free trastuzumab $(I \mu \mathrm{g} / \mathrm{mL})$ was used as positive control for HER2 binding. Data are mean $\pm S E(n=3)$. Statistical analysis was conducted using Wilcoxon-MannWhitney test, ${ }^{*}<0.05$ (B) Confocal laser-scanning micrographs of SKBR3 cells incubated for the indicated time points with PLGA-PEG-HC (green) and stained for HER2 (magenta). Nuclei are stained with DAPI (blue). Scale bar: $20 \mu \mathrm{m}$. (C) Time-course TEM images of PLGA-PEG-HC in SKBR3 cells. Arrows indicate endosomal compartments containing NPs. Scale bar: I $\mu \mathrm{m}$.

ulcerations with the reparative process of mucosal healing (Figure 3F, upper panels). Bowel lesions showed marked overexpression of MAdCAM-1 on the endothelium of mucosal vessels, while uninvolved colon tracts only displayed very few positive vessels (Figure $3 \mathrm{~F}$, lower panels), thus confirming the relevance of MAdCAM-1 as an IBD biomarker in this murine model. No mortality was observed in the DSS-treated mice.

\section{Detecting Colitis in Murine Model}

The capability of P@QD-MdC NPs to trigger fluorescent QDs at the site of bowel lesion was tested over time upon administration in a DSS-induced murine model of colitis and compared to NPs conjugated with aspecific immunoglobulin (P@QD-IgG) or bare NPs (P@QD). After intravenous injection, all nanocompounds showed a similar circulation profile in the bloodstream of mice (Supplementary Figure 7). At 10 min, 1, 6 and 24 h post-injection, localization of NPs in the colon was analyzed ex vivo through measurement of epifluorescence along the colon of treated mice (Figure 4A). Results showed that all nanocompounds were able to reach the colon of DSS-treated mice to a weak extent within $6 \mathrm{~h}$ post-intravenous injection. However, only P@QD-MdC NPs showed a progressive increase in colon accumulation over time, while P@QD-IgG and P@QD were mostly associated with a decrease of fluorescence between 6 and $24 \mathrm{~h}$ post-injection. In particular, epifluorescence measured at $24 \mathrm{~h}$ post-injection was significantly higher in the colon of mice treated with P@QD-MdC as compared to P@QD-IgG $(p=0.030)$ and P@QD $(p=0.035$, Figure $4 \mathrm{~B}$ ), thus suggesting that MAdCAM-1 targeting triggered a site-specific binding of the NPs at IBD foci and achieved specific imaging of the inflamed bowel. The injection of either P@QD-MdC, P@QD-IgG or P@QD had no impact on colon length in DSS-treated mice, which appeared equal to that of mice injected with vehicle only (Figure 4C). MAdCAM-1 was equally expressed in the inflamed colon in all groups of mice (Figure 4D).

Evidence of P@QD-MdC NPs stacking on the endothelium of bowel mucosal vessels was assessed by electron microscopy. SEM imaging, carried out upon metal-coating of colon specimens obtained at $24 \mathrm{~h}$ postNP-injection, showed the presence of spherical structures lying on the endothelium of bowel veins (Figure 5). Size 
A

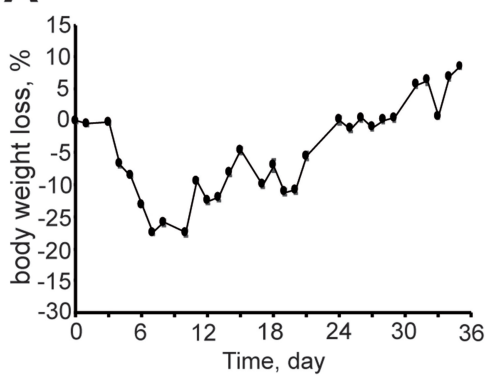

D

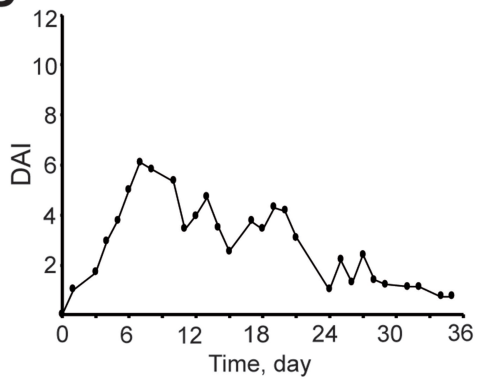

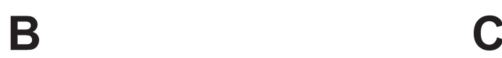
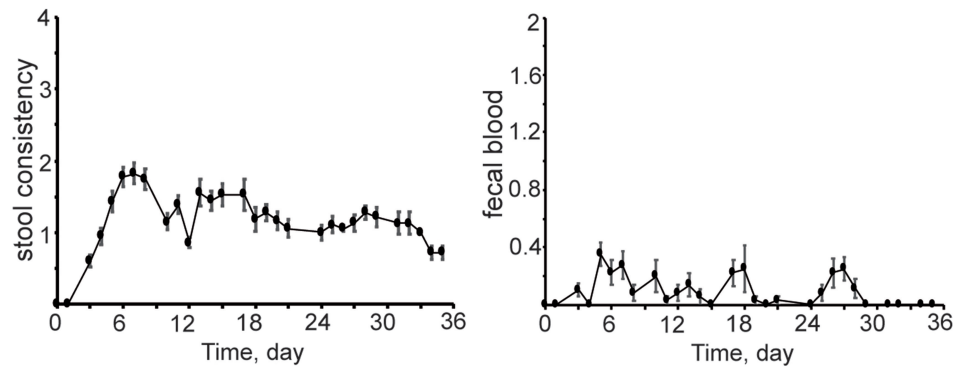

E

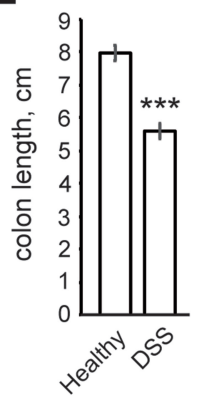

$\mathbf{F}$

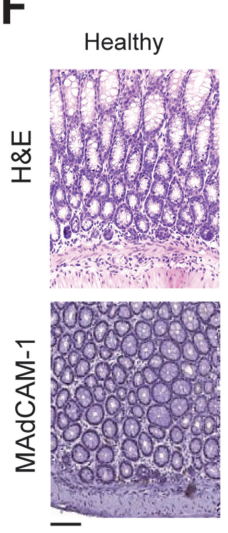

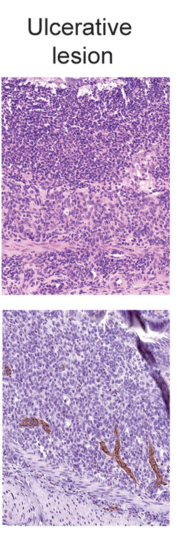

Reparative lesion

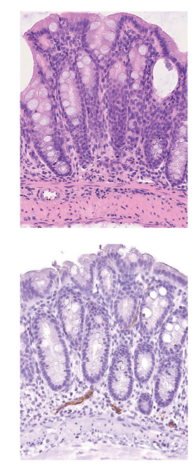

Figure 3 Murine model of chronic colitis obtained through DSS treatment. (A) Body weight change, (B) stool consistency, (C) fecal blood and (D) disease activity index (DAI) were scored daily over 3 cycles of DSS treatment. Results are mean \pm SE $(n=18)$. (E) Colon length of healthy untreated mice and DSS-treated mice was measured at the end of treatment (day 36). Results are mean \pm SE $(n=18)$. Statistical analysis was conducted using Student's $t$-test, $* * * p<0.000 I$. (F) Hematoxylin and eosin staining $(H \& E)$ of three representative colon sections from DSS-treated mice (upper panels) and corresponding MAdCAM-I immunohistochemistry (lower panels) (20× magnification). Scale bar: I00 $\mu$ m.

and shape of the structures reflected those of P@QD-MdC NPs (Supplementary Figure 8). As control, mice treated with vehicle were equally analyzed by SEM and none of these structures were observed.

Upon NP treatment, the histological assessment of bowel damage confirmed the chronic nature of colon inflammation, with regions of epithelial regeneration, mild-to-moderate inflammatory infiltrate and some ulcerative epithelial changes from previous acute damaging events (Figure 6A-C). Signs of reduced inflammation were observed in mice treated with $\mathrm{P} @$ QD-MdC, where epithelial regeneration was predominant over the whole colon, while mice treated with P@QD-IgG and P@QD NPs still displayed more severe mucosal inflammatory infiltrates (Figure 6D and Supplementary Table 1).

\section{Systemic Biodistribution}

Systemic biodistribution revealed that NPs were mainly captured by the liver. NPs signal was also high in lungs and spleen at $10 \mathrm{~min}$ and $1 \mathrm{~h}$ post-injection, while lower levels of epifluorescence were measured from $10 \mathrm{~min}$ to $24 \mathrm{~h}$ in brain, kidneys, small bowel and heart, thus supporting differential accumulation of NPs upon i.v. injection (Figure 7A and B). Transit kinetics of NPs revealed a significant washout from the liver between 1 and $24 \mathrm{~h}$ post-injection (mean Delta $\operatorname{Epf}_{(24 \mathrm{~h}-1 \mathrm{~h})}=-76.8 \pm$ $13.5 \%, \mathrm{p}=0.01)$. A trend toward reduction was also assessed in lungs (mean Delta $\operatorname{Epf}_{(24 \mathrm{~h}-1 \mathrm{~h})}=-92.8 \pm$ $1.9 \%, \mathrm{p}=0.06)$ and spleen (mean Delta $\operatorname{Epf}_{(24 \mathrm{~h}-1 \mathrm{~h})}=$ $-67.1 \pm 16.1 \%, p=0.08)$, thus supporting a progressive washout of the nanocompounds from the main off-target organs (Supplementary Figure 9).

Histological assessment of dissected organs showed that no lesions were induced by NP administration (Figure 7C). Moreover, no signs of toxicity (body weight loss, pain or discomfort) and no reactions were observed following NP administration at the injection site, thus supporting the overall safety of P@QD-MdC.

\section{Nanodetection of IBD in Human Bowel Samples}

To further test the translational potential of P@QD-MdC for specific detection of diseased bowel segments, a preliminary experiment was conducted ex vivo on bowel 

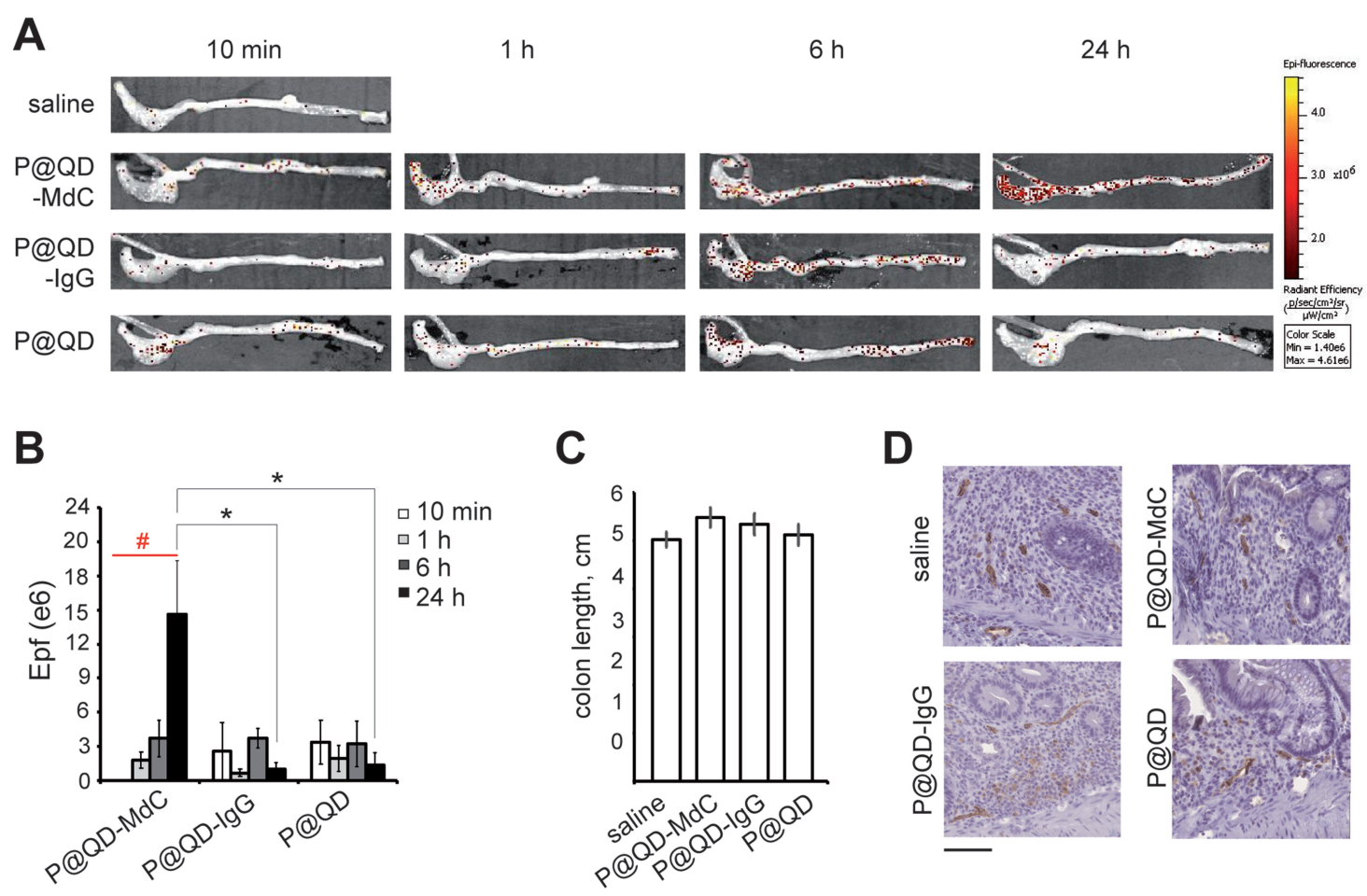

Figure 4 P@QD-MdC NPs target colitis in a murine model. (A) Epifluorescence (EPf) images of representative colons dissected from mice exposed to saline, P@QD-MdC, P@QD-IgG or P@QD at different time points. Epf is expressed as radiant efficiency $\left(\mathrm{p} / \mathrm{sec} / \mathrm{cm}^{2} / \mathrm{sr}\right) /\left(\mathrm{W} / \mathrm{cm}^{2}\right)$. (B) Epf intensity of colons dissected at $10 \mathrm{~min}, \mathrm{I}, 6,24 \mathrm{~h}$ postNP-injection. Results are mean \pm SE $(n=5)$. Comparison between NPs at each time point was conducted using Wilcoxon-Mann-Whitney test, *p<0.05 P@QD-MdC vs P@QD-IgG and P@QD at $24 \mathrm{~h}$. Trend over time for each type of NP was analyzed by Kruskal-Wallis test, \#p=0.003 for P@QD-MdC. (C) Length of colon from mice exposed to saline or NPs for $24 \mathrm{~h}$. Results are mean \pm SE $(n=5)$. No statistical significance was observed by Kruskal-Wallis test $(p=0.5 \mathrm{I})$. (D) MAdCAM-I immunohistochemistry of representative colon sections from mice exposed to saline or NPs for $24 \mathrm{~h}$ (20x magnification). Scale bar: $100 \mu \mathrm{m}$.

tissue samples collected from patients with Crohn's Disease. PLGA-PEG-Mal NPs were loaded with QDs and conjugated with half-chains of a human MAdCAM-1 antibody. Incubated ex vivo on human bowel cryosections, the NPs provided fluorescent imaging of the mucosal microvasculature in the diseased bowel regions, while healthy tissues were only slightly stained by NPs (Figure 8A). The QD fluorescence intensity was significantly higher in the diseased bowel regions than in healthy bowel cryosections $(\mathrm{p}<0.0001$, Figure $8 \mathrm{~B})$, thus supporting the specificity of NPs for bowel lesions. Mucosal over-expression of MAdCAM-1 was confirmed in the corresponding diseased regions, while healthy functional bowel from matched patients showed very few MAdCAM-1-positive vessels (Figure 8C). To further characterize NP localization in diseased bowel specimens, a co-staining with anti-MAdCAM -1 antibody was performed on NP-exposed cryosections. Confocal microscopy revealed that NPs mostly localized on MAdCAM-1-positive vessels in the bowel mucosa of Crohn's Disease patients (Figure 8D), further supporting the molecular specificity of P@QD-MdC.

\section{MRI}

Once assessed the specificity of P@QD-MdC for inflamed bowel regions, we wished to step further and provide preliminary investigation on whether MAdCAM-1-targeted NPs could be useful for the development of a novel imaging tool by MRI. To this purpose, QDs were replaced by $\mathrm{MnO}$ nanocrystals, which were previously explored as safe and effective positive contrast enhancers in MRI. ${ }^{27,37}$ We recently demonstrated the colloidal stability of MnO-trapped PLGA-PEG NPs (MnO@PLGA) and measured their relaxivity potential in vitro, showing a dose and time-dependent $r_{1}$ enhancement caused by the release of $\mathrm{Mn}^{2+}$ ions from PLGA matrix. ${ }^{27}$ In this study, MnO@PLGA were tagged with anti-MAdCAM-1 halfchain portions and injected in mice affected by DSSinduced colitis. MRI scan of the rectum was performed at $24 \mathrm{~h}$ post-injection and revealed localized contrast enhanced regions following NP injection (Supplementary Figure 10). The T1-weighted images showed a selective enhancement of the mucosal layer in the rectum of treated mice, as result of the specific localization of functionalized NPs on MAdCAM-1-positive vessels. In comparison with 


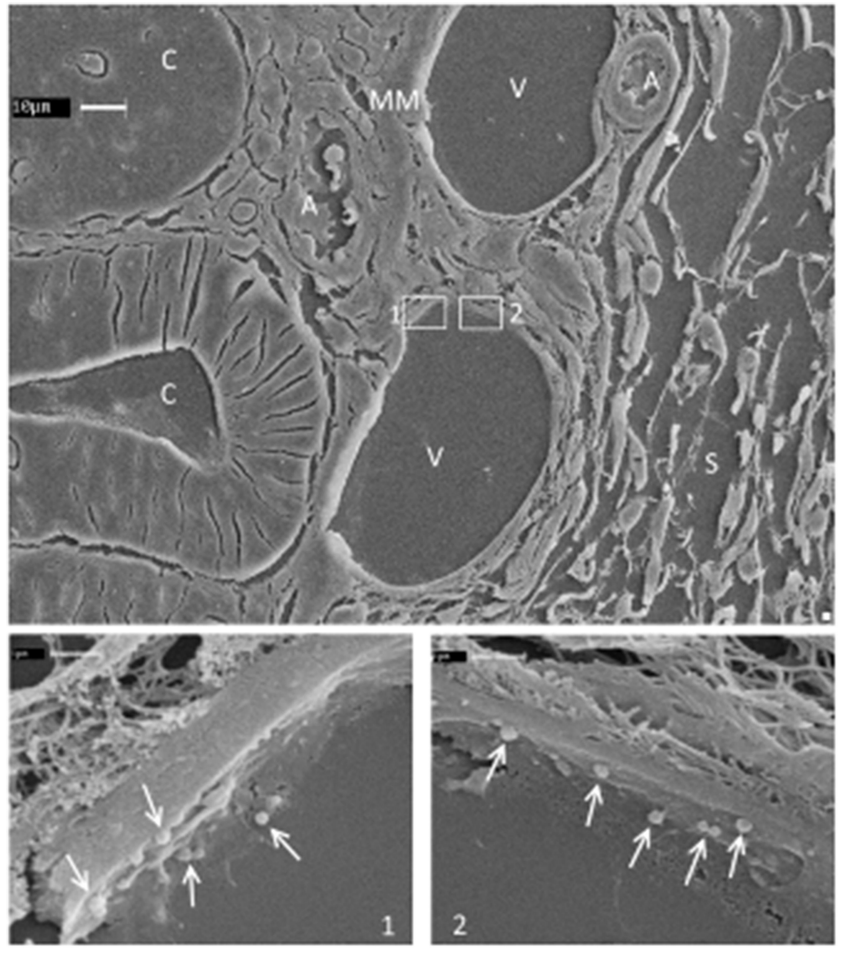

Figure 5 Representative SEM image of semi-thin sections of colon specimen obtained after $24 \mathrm{~h}$ treatment with P@QD-MdC NPs. Tissue structures are indicated with capital letters. High magnification of venous endothelial cells is shown in the square boxes. Arrows indicate roundish NPs on the surface of the endothelial cells. $C=$ crypt,$V=$ vein, $A=$ artery, $S=$ submucosa, $M M=$ muscularis mucosae, $L=$ lamina propria, $M E=$ muscularis externa.

non-contrast-enhanced images, the $\mathrm{T} 1$ images obtained upon NP injection showed contrast enhancement with correct maintenance of the anatomic background, which is a remarkable advantage for a device to be used as contrast agent.

\section{Discussion}

Currently, a major challenge for the clinical management of IBD is the lack of a method to specifically detect IBD foci at an early stage, as a sign of disease relapse and/or therapy failure. Due to the chronic nature of the disease, IBD patients frequently undergo a complex follow-up process consisting of invasive and uncomfortable endoscopic exams, aspecific resonance imaging and bowel ultrasound which, however, do not yield objective and precise information. ${ }^{5}$ A prompt and specific recognition of the disease activity status is of paramount importance as it could enable clinicians to select more appropriate therapies, monitor patients' responsiveness and avoid expensive and toxic treatments. In this context, a sensitive and tunable imaging device able to specifically trace early disease biomarkers could provide an accurate mapping of the diseased bowel and yield valuable information on the response to therapy or relapse of the disease.

The approach here presented is based on PLGA-PEG NPs modified by functionalization with half-chain portions of a monoclonal antibody directed toward MAdCAM-1 to form a stable and tunable nanodevice for IBD imaging. Through MAdCAM-1-targeted nanotechnology, we wished to mimic what happens to circulating leukocytes during inflammation. Once in the bloodstream, specific binding to MAdCAM-1 protein on the apical side of mucosal endothelial vessels is expected to promote stacking and penetration of NPs in the regions of bowel mucosa most affected by inflammation, where MAdCAM-1 is overexpressed. By coupling MAdCAM-1 targeting to an effective load of appropriate imaging agents (here proof of principle was demonstrated with QDs), we succeeded in obtaining specific detection of bowel inflammation, as the engineered NPs enabled release of the cargo molecules specifically at those bowel sites with MAdCAM-1 overexpression.

The capability of conjugated NPs to mediate effective and specific molecular targeting was studied in vitro using a model antibody for biomedical applications. Analysis of cellular interaction showed excellent recognition of the target protein and functionalization for selective targeting. Biodegradation of the organic component of the NPs was documented by the progressive rupture of the polymeric micelle in the intracellular compartment. Then, the capability of P@QD-MdC NPs to specifically detect bowel inflammation was tested in a murine model of chronic colitis obtained by oral treatment with DSS. P@QDMdC NPs progressively accumulated in the colon of mice and provided an enhanced and localized signal with respect to control untargeted NPs. NPs were visualized by SEM, which provided direct evidence of specific localization on the apical face of mucosal endothelial vessels. To our knowledge, this is the first report indicating the potential of SEM imaging to capture PLGA nano-localization in the gut with unprecedented precision.

Treatment of mice with P@QD-MdC NPs appeared to induce bowel remodeling and reduce severity of the inflammatory infiltrate as compared to control NPs. Despite gathered from a reduced number of animals, this inceptive observation could be of relevance if we consider that human anti-MAdCAM-1 antibody (SHP647) has shown encouraging results in reducing lymphocyte homing to the intestinal tract in patients affected by IBD. The TURANDOT trial has shown that blocking MAdCAM-1 
A

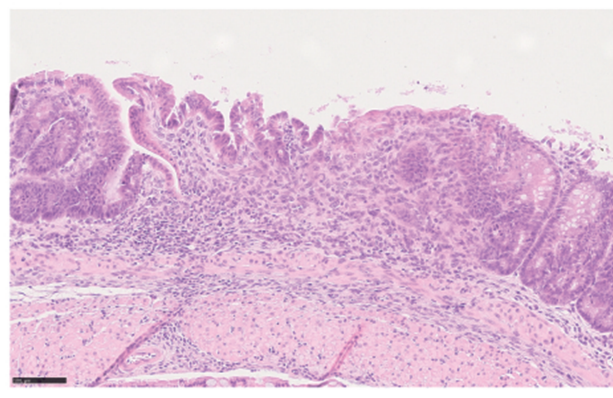

B

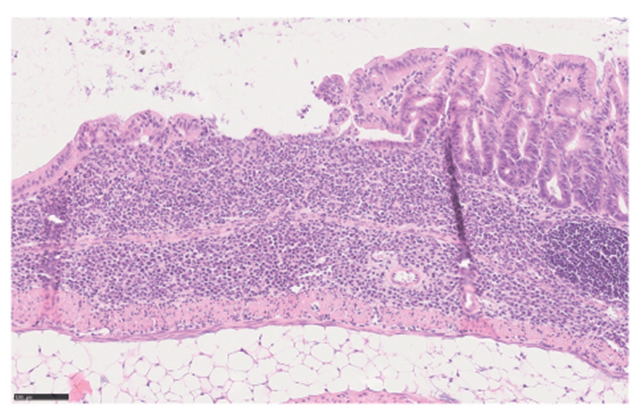

C

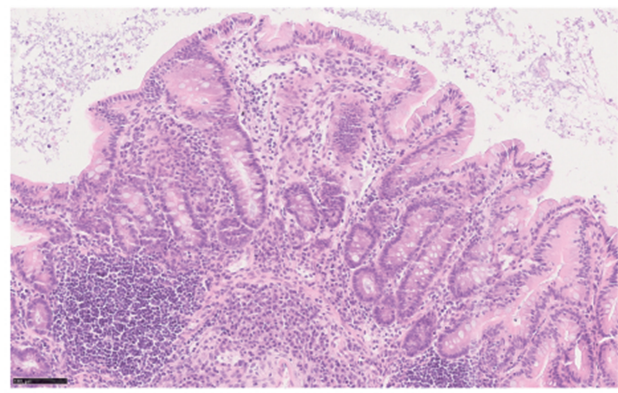

D

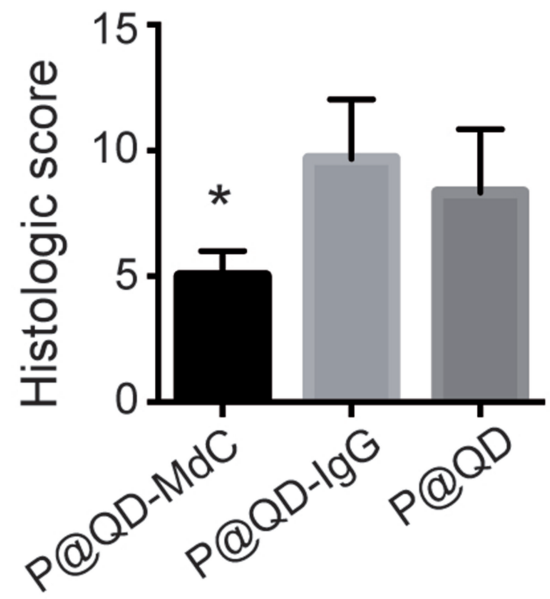

Figure 6 Colon damage assessed by histology. Representative images (20x magnification) of mild colonic inflammation and active epithelial regeneration (A); bowel ulceration and severe inflammatory infiltrate (B); and moderate inflammatory infiltrate in the glandular epithelium with no sign of erosion nor regeneration (C). Scale bar: $100 \mu \mathrm{m}$. (D) Histologic score of mice treated with NPs for $24 \mathrm{~h}$, measured as the sum of inflammation and epithelial changes as described in the Methods section. Data are mean \pm SD $(n=3)$. Statistical analysis was conducted by Wilcoxon-Mann-Whitney test ( $90 \%$ confidence level), *P=0.05 P@QD-MdC vs P@QDIgG and P@QD.

may induce remission in moderate-to-severe Ulcerative Colitis and a human monoclonal anti-MAdCAM-1 antibody is now under phase III evaluation. ${ }^{14,18}$ Our engineered NPs are equipped with the half-chain portions rather than the entire anti-MAdCAM-1 antibody, but still achieve specific binding to the molecular target. Whether and to what extent they may block MAdCAM-1 and hamper leukocyte extravasation is still unexplored but would deserve further investigation in appropriate and long-term treatment settings.

P@QD-MdC NPs were also tested ex vivo in human bowel surgical samples from patients with Crohn's Disease. Reported specificity for the stenotic bowel further supported the translational potential of the as-designed NPs. Concordance between NP accumulation and MAdCAM-1 expression indicated selective targeting efficiency and strengthened the conclusion that MAdCAM-1-targeted
PLGA-PEG NP could be a promising candidate for development as a nano-imaging agent for specific IBD detection.

This study contributes to the growing body of evidence that MAdCAM-1 merits attention as an IBD biomarker, and that it may be suitable as a molecular target for appropriately engineered NPs. PLGA-PEG NPs decorated with multiple anti-MAdCAM-1 half-chains are a promising tool to obtain specific image-guided detection of IBD location and severity.

Beyond the colon, MAdCAM-1 is known to be expressed in the Purkinje cells of the cerebellum. ${ }^{38}$ In our murine study, no preferential accumulation of P@QDMdC NPs was noticed in the brain. This observation could be attributed to the fact that large NPs hardly pass the blood-brain barrier, which remains impermeable to most of the drugs and nanodrugs administered. Size and negative charge of our NPs could in part explain why they did 
A

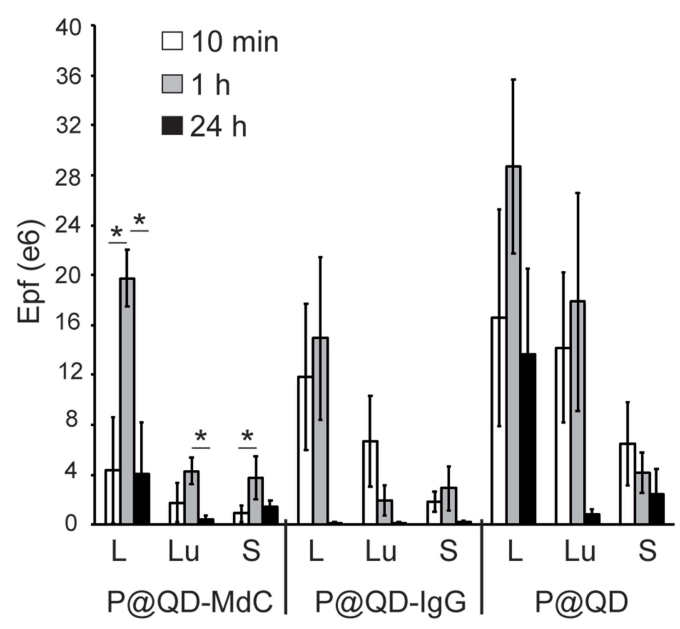

B

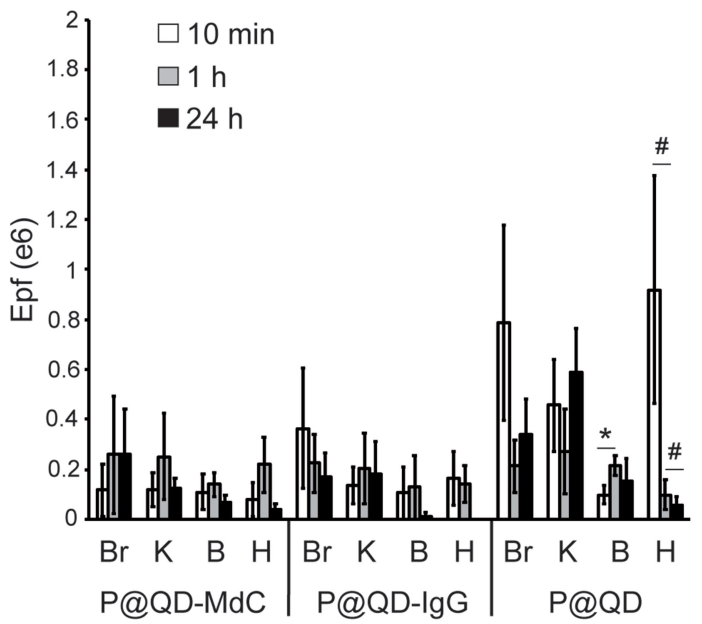

C P@QD-MdC P@QD-IgG P@QD
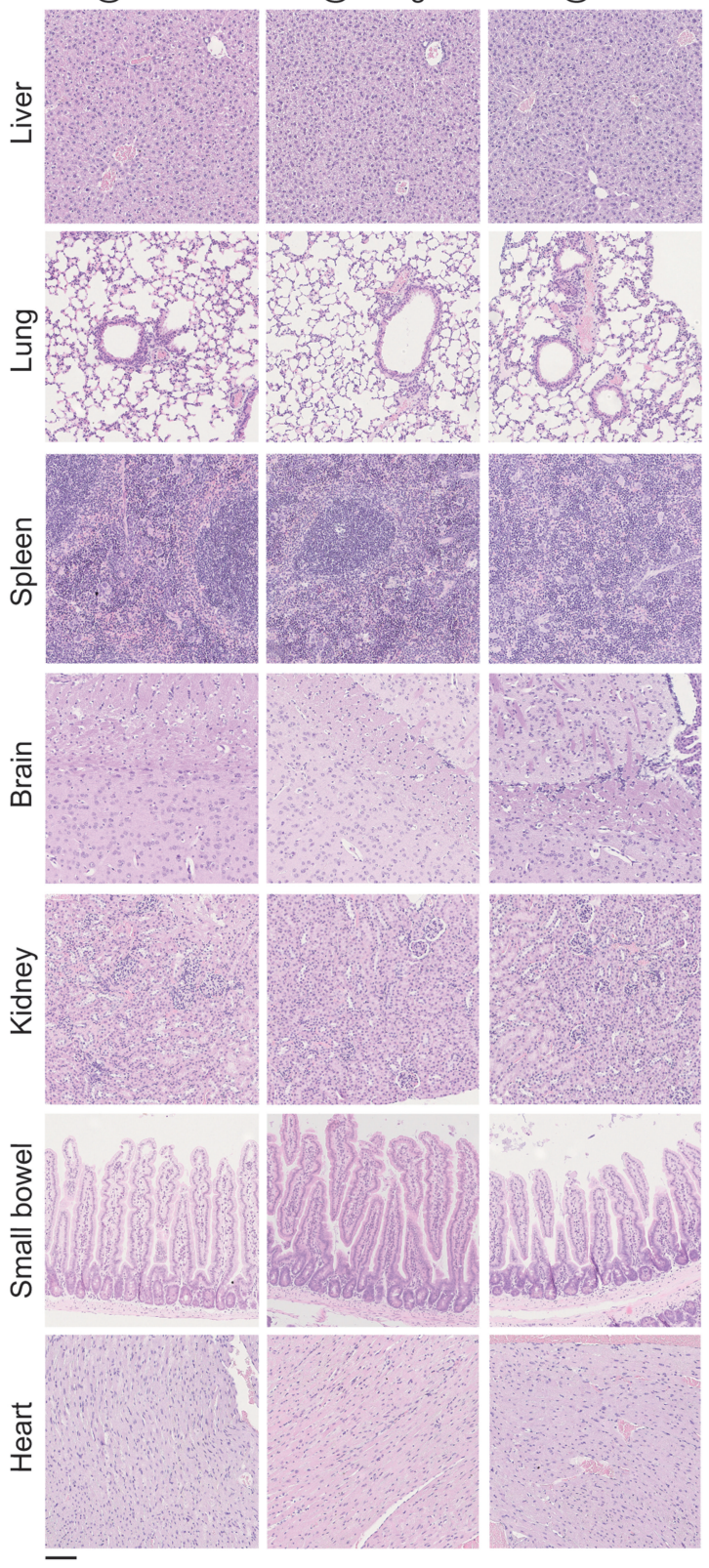

Figure 7 Systemic biodistribution of NPs as assessed by fluorescence measurement of excised organs. Average epifluorescence (Epf) intensity of liver (L), lungs (Lu), spleen $(\mathrm{S})(\mathbf{A})$ or brain $(\mathrm{Br})$, kidneys $(\mathrm{K})$, small bowel $(\mathrm{B})$ and heart $(\mathrm{H})(\mathbf{B})$ at $10 \mathrm{~min}, \mathrm{I}, 24 \mathrm{~h}$ post-NP-injection. Epf is expressed as radiant efficiency $\left(\mathrm{p} / \mathrm{sec} / \mathrm{cm}^{2} / \mathrm{sr}\right) /\left(\mu \mathrm{W} / \mathrm{cm}^{2}\right)$. Results are mean \pm SE $(n=5)$. Statistical analysis was conducted by Wilcoxon-Mann-Whitney test $\left(90 \%\right.$ confidence level), ${ }^{*} \mathrm{p}=0.06$, ${ }^{\#} \mathrm{P}=0.09$. $(\mathbf{C}) \mathrm{H} \& \mathrm{E}$ of non-target organs from representative mice exposed to NPs for $24 \mathrm{~h}(20 \times$ magnification). Scale bar: $100 \mu \mathrm{m}$.

not preferentially accumulate in the brain of treated mice. Secondly, we only performed a single injection. The expression of MAdCAM-1 in the cerebellum may however become an important issue in case one could plan to increase the administered dosage or schedule repeated administrations.

This study has some limitations. In particular, we demonstrated the proof of efficacy in an ex vivo context and with the use of QDs fluorescence as the imaging read- out. We chose QDs, which is a stable and sensitive fluorescent tracer, as a means to track the nano-localization and to provide a proof of principle preclinical study for MAdCAM-1 targeting by PLGA-PEG NPs. Translational exploitation of these nanoconjugates will involve the replacement of QDs with appropriate contrast agents for MRI application. We have already synthesized manganese oxide $(\mathrm{MnO})$ NPs embedded in functional PLGA polymers and tested their potential as $\mathrm{T} 1$ contrast agents for 

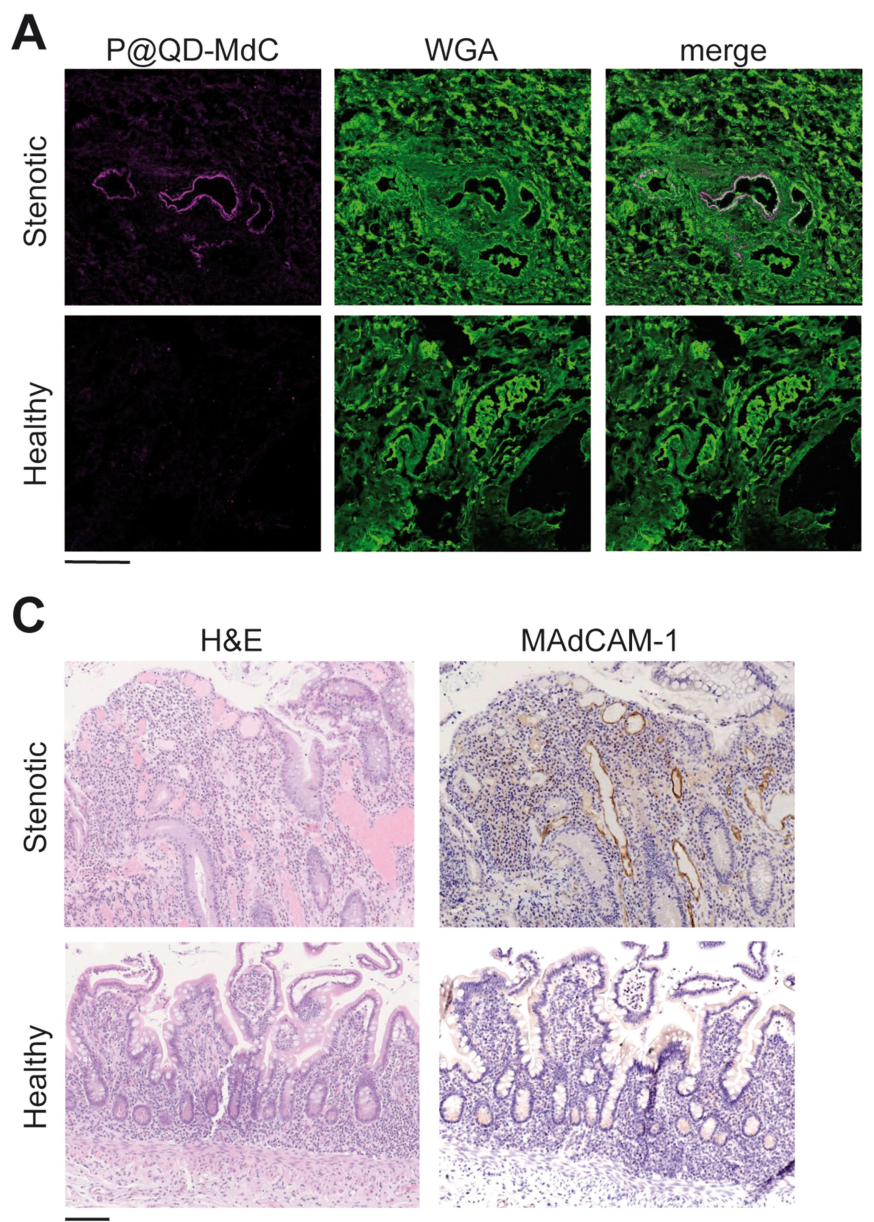

B

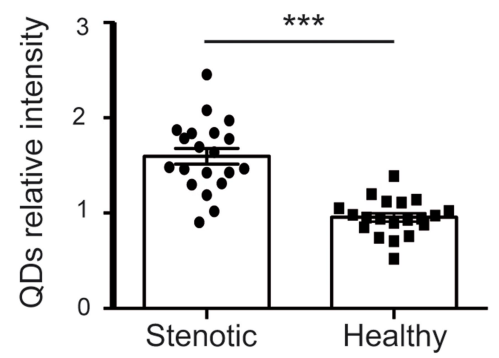

D
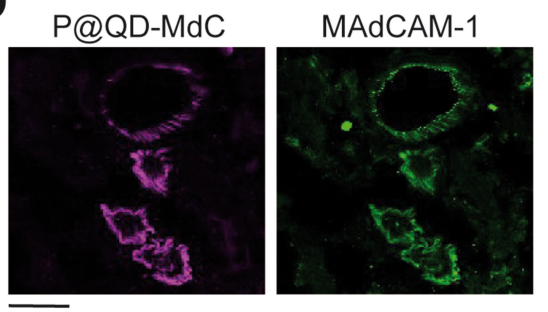

merge

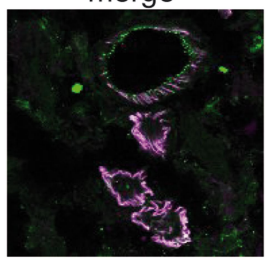

Figure 8 P@QD-MdC NPs detect IBD foci in human bowel specimens ex vivo. (A) Confocal laser scanning images of intestinal cryosections from stenotic or healthy bowel mucosa incubated with P@QD-MdC NPs (magenta). Cellular plasma membranes are stained with WGA (green). Scale bar: I00 $\mu$ m. (B) Quantification of QDs fluorescence intensity on stenotic and healthy bowel cryosections from 3 different patients. For each section, at least 6 fields of view were analyzed. Data shown are the mean \pm SE. Statistical analysis was conducted using Student's $t$-test, ${ }^{* * *} \mathrm{p}<0.000 \mathrm{I}$. (C) Hematoxylin and eosin staining (H\&E, left panels) and corresponding MAdCAM-I immunohistochemistry (right panels) of representative bowel sections from stenotic and healthy tract (20x magnification). Scale bar: 100 $\mu \mathrm{m}$ (D) Co-staining of P@QD-MdC NPs (magenta) and anti-MAdCAM-I antibody (green) on a representative stenotic bowel section. Scale bar: $50 \mu \mathrm{m}$.

MRI. $^{27}$ Here, the implementation of MnO@PLGA with MAdCAM-1 targeting provided preliminary evidence of targeted and effective in vivo imaging of the inflamed colon. Results obtained by MRI scan of the rectum revealed selective contrast enhancement of the bowel mucosa as shown by $\mathrm{T} 1$ images in colitic mice. Despite preliminary, this piece of data is sound if we consider that commercially available contrast agents distribute in various cellular compartments by non-specific uptake. ${ }^{39}$ By contrast, the bioengineered NPs are endowed with selective ligands for specific recognition of MAdCAM-1 protein, enabling more accurate mapping of the IBD foci. Dedicated studies are now required to determine appropriate dosage, time to washout and detailed toxicology aspects. However, this preliminary study provides proof of specificity and potential for a novel T1 contrast enhancer for IBD imaging. PLGA-PEG NPs targeted to MAdCAM-1 are expected to obtain reliable and IBDspecific signal enhancement detectable by abdominal MRI. This hypothesis has the potential to enable a more accurate, specific and non-invasive diagnosis of IBD, and warrants further investigation.

\section{Conclusion}

In this study, we provide evidence that active targeting of MAdCAM-1 by engineered PLGA-PEG NPs loaded with QDs enables specific imaging of IBD in an experimental murine model and bowel tissues from Crohn's Disease patients. Fine-tuning of this nanodevice with appropriate imaging agents holds potential for clinical translation and is expected to trigger an outstanding improvement for IBD diagnostic reliability as well as substantially impacting patients' quality of life, thanks 
to its non-invasive nature. The opportunity to specifically image IBD at an early stage of disease and with a safe, sensitive and molecularly targeted nanodevice may represent a significant breakthrough in the translational application of nanomedicine for a better clinical management of IBD.

\section{Abbreviations}

DAI, disease activity index; DLS, dynamic light scattering; DSS, dextran sulfate sodium salt; FDA, Food and Drug Administration; FTIR, Fourier-transform infrared spectroscopy; HC, half-chain; HER2, human epidermal growth factor 2; H\&E, hematoxylin and eosin staining; IBD, Inflammatory Bowel Disease; IgG, immunoglobulin; MAdCAM-1, mucosal addressin cell adhesion molecule-1; Mal, maleimide compound; $\mathrm{MnO}$, manganese oxide; MRI, magnetic resonance imaging; NP, nanoparticle; PBS, phosphate buffer saline; PEG, polyethylene glycol; PDI, polydispersity index; PLGA, poly(lactic-co-glycolic acid); QDs, quantum dots; SEM, scanning electron microscopy; TEM, transmission electron microscopy; TZ, trastuzumab; WGA, wheat germ agglutinin.

\section{Acknowledgments}

We thank the Pediatric Clinical Research Center "Romeo and Enrica Invernizzi" at Università degli studi di Milano for access to the imaging facility and for SM support. We also thank Dr. A. Esposito and T. Canu (IRCCS San Raffaele Hospital, Milano, Italy) for magnetic resonance service. We are grateful to Mrs. R. Allpress for the professional revision of English, and to Prof. M. Nebuloni and Dr. L. Villani for their helpful comments. We thank patients who participated in the study and all medical doctors at the IBD Surgery Division (ASST Fatebenefratelli Sacco) involved in patient care and research.

\section{Author Contributions}

All authors made a significant contribution to the work reported, whether that is in the conception, study design, execution, acquisition of data, analysis and interpretation, or in all these areas; took part in drafting, revising or critically reviewing the article; gave final approval of the version to be published; have agreed on the journal to which the article has been submitted; and agree to be accountable for all aspects of the work.

\section{Disclosure}

The authors have no conflict of interest to declare regarding this study.

\section{References}

1. Ananthakrishnan AN. Epidemiology and risk factors for IBD. Nat Rev Gastroenterol Hepatol. 2015;12:205-217.

2. Windsor JW, Kaplan GG. Evolving Epidemiology of IBD. Curr Gastroenterol Rep. 2019;21:40.

3. Ng SC, Shi HY, Hamidi N, et al. Worldwide incidence and prevalence of inflammatory bowel disease in the 21 st century: a systematic review of population-based studies. Lancet Lond. Engl. 2018;390:2769-2778. doi:10.1016/S0140-6736(17)32448-0

4. Pasvol TJ, Horsfall L, Bloom S, et al. Incidence and prevalence of inflammatory bowel disease in UK primary care: a population-based cohort study. BMJ Open. 2020;10:e036584.

5. Gomollón F, Dignass A, Annese V, et al. 3rd European evidencebased consensus on the diagnosis and management of Crohn's disease 2016: part 1: diagnosis and medical management. J Crohns Colitis. 2017;11:3-25.

6. Magro F, Doherty G, Peyrin-Biroulet L, et al. ECCO position paper: harmonisation of the approach to ulcerative colitis histopathology. J Crohns Colitis. 2020. doi:10.1093/ecco-jcc/jjaa110

7. Vegh Z, Burisch J, Pedersen N, et al. Incidence and initial disease course of inflammatory bowel diseases in 2011 in Europe and Australia: results of the 2011 ECCO-EpiCom inception cohort. J Crohns Colitis. 2014;8:1506-1515.

8. Rieder F, Latella G, Magro F, et al. European Crohn's and colitis organisation topical review on prediction, diagnosis and management of fibrostenosing Crohn's disease. J Crohns Colitis. 2016;10 (8):873-885. doi:10.1093/ecco-jcc/jjw055

9. Van Assche G, Dignass A, Reinisch W, et al. The second European evidence-based consensus on the diagnosis and management of Crohn's disease: special situations. J Crohns Colitis. 2010;4:63-101.

10. Horsthuis K, Bipat S, Bennink RJ, Stoker J. Inflammatory bowel disease diagnosed with US, MR, scintigraphy, and CT: meta-analysis of prospective studies. Radiology. 2008;247:64-79. doi:10.1148/radiol.2471070611

11. Lönnfors S, Vermeire S, Greco M, et al. IBD and health-related quality of life - discovering the true impact. J Crohns Colitis. 2014;8:1281-1286. doi:10.1016/j.crohns.2014.03.005

12. Xavier RJ, Podolsky DK. Unravelling the pathogenesis of inflammatory bowel disease. Nature. 2007;448:427-434. doi:10.1038/nature06005

13. Zhang Y-Z, Li -Y-Y. Inflammatory bowel disease: pathogenesis. World J Gastroenterol. 2014;20:91-99. doi:10.3748/wjg.v20.i1.91

14. Vermeire S, Sandborn WJ, Danese S, et al. Anti-MAdCAM antibody (PF-00547659) for ulcerative colitis (TURANDOT): a Phase 2, randomised, double-blind, placebo-controlled trial. Lancet Lond. Engl. 2017;390:135-144. doi:10.1016/S0140-6736(17)30930-3

15. Allocca M, Gilardi D, Fiorino G, et al. PF-00547659 for the treatment of Crohn's disease and ulcerative colitis. Expert Opin Investig Drugs. 2018;27:623-629. doi:10.1080/13543784.2018.1494722

16. Neurath MF. New targets for mucosal healing and therapy in inflammatory bowel diseases. Mucosal Immunol. 2014;7:6-19.

17. Reinisch W, Hung K, Hassan-Zahraee M, Cataldi F. Targeting endothelial ligands: ICAM-1/alicaforsen, MAdCAM-1. J Crohns Colitis. 2018;12:S669-S677. doi:10.1093/ecco-jcc/jjy059

18. Duijvestein M, D'Haens GR. Rational and clinical development of the anti-MAdCAM monoclonal antibody for the treatment of IBD. Expert Opin Biol Ther. 2019;19:361-366. doi:10.1080/ 14712598.2019.1576631

19. Sands BE, Feagan BG, Rutgeerts P, et al. Effects of vedolizumab induction therapy for patients with Crohn's disease in whom tumor necrosis factor antagonist treatment failed. Gastroenterology. 2014;147:618-627.e3. doi:10.1053/j.gastro.2014.05.008

20. Feagan BG, Rubin DT, Danese S, et al. Efficacy of vedolizumab induction and maintenance therapy in patients with ulcerative colitis, regardless of prior exposure to TNF antagonists. Clin Gastroenterol Hepatol. 2016. doi:10.1016/j.cgh.2016.08.044 
21. Singh H, Grewal N, Arora E, Kumar H, Kakkar AK. Vedolizumab: A novel anti-integrin drug for treatment of inflammatory bowel disease. J Nat Sci Biol Med. 2016;7:4-9. doi:10.4103/0976-9668.175016

22. Truffi M, Colombo M, Peñaranda-Avila J, et al. Nano-targeting of mucosal addressin cell adhesion molecule-1 identifies bowel inflammation foci in murine model. Nanomed. 2017;12:1547-1560. doi:10.2217/nnm-2017-0004

23. Bachmann C, Klibanov AL, Olson TS, et al. Targeting mucosal addressin cellular adhesion molecule (MAdCAM)-1 to noninvasively image experimental Crohn's disease. Gastroenterology. 2006;130:8-16. doi:10.1053/j.gastro.2005.11.009

24. Lü J-M, Wang X, Marin-Muller C, et al. Current advances in research and clinical applications of PLGA-based nanotechnology. Expert Rev Mol Diagn. 2009;9:325-341. doi:10.1586/erm.09.15

25. Elmowafy EM, Tiboni M, Soliman ME. Biocompatibility, biodegradation and biomedical applications of poly(lactic acid)/poly(lactic-coglycolic acid) micro and nanoparticles. $J$ Pharm Investig. 2019;49:347-380. doi:10.1007/s40005-019-00439-x

26. El-Hammadi MM, Arias JL. Advanced engineering approaches in the development of PLGA-based nanomedicines. In: Aliofkhazraei M, editor. Handbook of Nanoparticles. Springer, Cham; 2016. doi:10.1007/978-3-319-15338-4_45

27. Mauri M, Collico V, Morelli L, et al. MnO nanoparticles embedded in functional polymers as $\mathrm{T} 1$ contrast agents for magnetic resonance imaging. ACS Appl Nano Mater. 2020;3(4):3787-3797. doi:10.1021/ acsanm.0c00474

28. Morelli L, Gimondi S, Sevieri M, et al. Monitoring the fate of orally administered PLGA nanoformulation for local delivery of therapeutic drugs. Pharmaceutics. 2019;11:658. doi:10.3390/pharmaceutics11120 658

29. Chan JM, Valencia PM, Zhang L, Langer R, Farokhzad OC. Polymeric nanoparticles for drug delivery. Methods Mol Biol Clifton NJ. 2010;624:163-175.
30. Cheng J, Teply B, Sherifi I, et al. Formulation of functionalized PLGA-PEG nanoparticles for in vivo targeted drug delivery. Biomaterials. 2007;28:869-876. doi:10.1016/j.biomaterials.2006.09.047

31. Truffi M, Colombo M, Sorrentino L, et al. Multivalent exposure of trastuzumab on iron oxide nanoparticles improves antitumor potential and reduces resistance in HER2-positive breast cancer cells. Sci. Rep. 2018;8:6563. doi:10.1038/s41598-018-24968-X

32. Di Gregorio J, Sferra R, Speca S, et al. Role of glycogen synthase kinase-3 $\beta$ and PPAR- $\gamma$ on epithelial-to-mesenchymal transition in DSS-induced colorectal fibrosis. PLoS One. 2017;12:e0171093.

33. Vetrano S, Rescigno M, Rosaria Cera M, et al. Unique role of junctional adhesion molecule-a in maintaining mucosal homeostasis in inflammatory bowel disease. Gastroenterology. 2008;135:173-184. doi:10.1053/j.gastro.2008.04.002

34. Farkas S, Hornung M, Sattler C, et al. Blocking MAdCAM-1 in vivo reduces leukocyte extravasation and reverses chronic inflammation in experimental colitis. Int $J$ Colorectal Dis. 2006;21:71-78. doi:10.1007/s00384-004-0709-y

35. Obermeier F, Dunger N, Strauch UG, et al. Contrasting activity of cytosin-guanosin dinucleotide oligonucleotides in mice with experimental colitis. Clin Exp Immunol. 2003;134:217-224. doi:10.1046/ j.1365-2249.2003.02288.x

36. Song X, Zhao Y, Hou S, et al. Dual agents loaded PLGA nanoparticles: systematic study of particle size and drug entrapment efficiency. Eur J Pharm Biopharm. 2008;69:445-453.

37. Na HB, Lee JH, An K, et al. Development of a T1 contrast agent for magnetic resonance imaging using $\mathrm{MnO}$ nanoparticles. Angew Chem Int Ed Engl. 2007;46:5397-5401.

38. Human protein atlas. Available from: http://www.proteinatlas.org. Accessed October 10, 2020.

39. Aime S, Caravan P. Biodistribution of gadolinium-based contrast agents, including gadolinium deposition. J Magn Reson Imaging. 2009;30:1259-1267.
International Journal of Nanomedicine

\section{Publish your work in this journal}

The International Journal of Nanomedicine is an international, peerreviewed journal focusing on the application of nanotechnology in diagnostics, therapeutics, and drug delivery systems throughout the biomedical field. This journal is indexed on PubMed Central, MedLine, CAS, SciSearch ${ }^{\circledR}$, Current Contents ${ }^{\mathbb{R}} /$ Clinical Medicine,

\section{Dovepress}

Journal Citation Reports/Science Edition, EMBase, Scopus and the Elsevier Bibliographic databases. The manuscript management system is completely online and includes a very quick and fair peer-review system, which is all easy to use. Visit http://www.dovepress.com/ testimonials.php to read real quotes from published authors. 